\title{
Structure and binding in crystals of cagelike molecules: Hexamine and platonic hydrocarbons
}

\author{
Kristian Berland ${ }^{\text {a) }}$ and Per Hyldgaard \\ Department of Microtechnology and Nanoscience, MC2, Chalmers University of Technology, \\ SE-41296 Göteborg, Sweden
}

(Received 28 October 2009; accepted 25 February 2010; published online 6 April 2010)

\begin{abstract}
In this paper, we show that first-principle calculations using a van der Waals density functional (vdW-DF) [M. Dion, H. Rydberg, E. Schröder, D. C. Langreth, and B. I. Lundqvist, Phys. Rev. Lett. 92, 246401 (2004)] permit the determination of molecular crystal structure within density functional theory (DFT). We study the crystal structures of hexamine and the platonic hydrocarbons (cubane and dodecahedrane). The calculated lattice parameters and cohesion energy agree well with experiments. Further, we examine the asymptotic accounts of the van der Waals forces by comparing full vdW-DF with asymptotic atom-based pair potentials extracted from vdW-DF. The character of the binding differs in the two cases, with vdW-DF giving a significant enhancement at intermediate and relevant binding separations. We analyze consequences of this result for methods such as DFT-D and question DFT-D's transferability over the full range of separations. (C) 2010 American Institute of Physics. [doi:10.1063/1.3366652]
\end{abstract}

\section{INTRODUCTION}

Understanding supramolecular structure and interactions is essential for understanding many biological processes. ${ }^{1}$ Biological and other supramolecular complexes as polymers and overlayers are sparse matter, that is, they contain low electronic density in essential regions. The general lack of order in these systems prohibits precise measurements of atomic structure and therefore challenges development of theoretical methods. In turn, this makes transferable firstprinciple schemes attractive; an accurate account of simple periodic structures permits accurate characterization to be made and reliable conclusions to be drawn for more complex (nonperiodic) systems. Molecular crystals and simple polymer crystals ${ }^{2}$ are ideal testing grounds for applications of first-principle descriptions of sparse matter. Unlike most sparse matter, they constitute ordered systems and therefore lead to unambiguous comparison between theory and experiments.

The dispersion forces underpin the cohesion of sparse matter. Modeling of sparse matter at the electronic level therefore requires that we take these effects into account. Traditional implementations of density functional theory (DFT), which parametrizes the density functional within the local-density approximation (LDA) (Ref. 3) or the generalized gradient approximation (GGA) (Refs. 4 and 5), have allowed for routine modeling of matter with dense electron distributions. However, the lack of nonlocal correlation and hence dispersion forces in these implementations has effectively inhibited widespread use for general sparse matter systems. Computational methods able to predict molecular crystal structure and stability are also of value in the pharmaceutical industry, for example, in mapping out competing crystalline phases for the fabrication of

${ }^{a)}$ Electronic mail: berland@chalmers.se. pharmaceuticals. ${ }^{6}$ The electronic structure and response are often required to understand and compute properties of technological significance. Consequentially, a range of developments has aimed to extend density functional approximations with an account of dispersive interactions and thus capability to address sparse matter challenges. Such approaches include nonlocal functionals, ${ }^{7-12}$ intermolecular perturbation theory, ${ }^{13,14}$ and the addition of a semiemperical atom-based account of the dispersion forces (DFT-D). ${ }^{15-18}$ DFT-D has also been used to characterize molecular crystals. ${ }^{19,20}$

We study molecular crystal using a van der Waals density functional (vdW-DF). ${ }^{10}$ The correlation part of this functional depends nonlocally on the density and accounts for dispersion forces. vdW-DF has been used in a series of firstprinciple studies of sparse matter, ${ }^{21}$ yielding, for example, new insight to the twist of DNA, ${ }^{22}$ nanotube bundles, ${ }^{23}$ water hexamers, ${ }^{24}$ metal-organic frameworks, ${ }^{25}$ and the binding mechanisms at organic metal interfaces. ${ }^{26-29}$

In this paper, we first demonstrate that vdW-DF permits structural determination of molecular crystals and second assess the character of the attractive dispersion interactions. For the first, we calculate the lattice parameters for crystals of the cagelike hexamine, dodecahedrane, and cubane molecules. For the second, we compare an atom-centered asymptotic- $1 / r^{6}$ approximation frequently encountered in force-field methods ${ }^{6,30,31}$ and in DFT-D, with the correlation energy provided by vdW-DF. We also analyze consequences for DFT-D and question its performance at intermediate separations. The particular choice of molecules was motivated in part by their aesthetic appeal and in part for the property that their symmetric geometries permit a simple assignment and analysis of the strength of the asymptotic interactions even when expressed in terms of an atom-pair basis.

This paper has the following plan. The next section pre- 

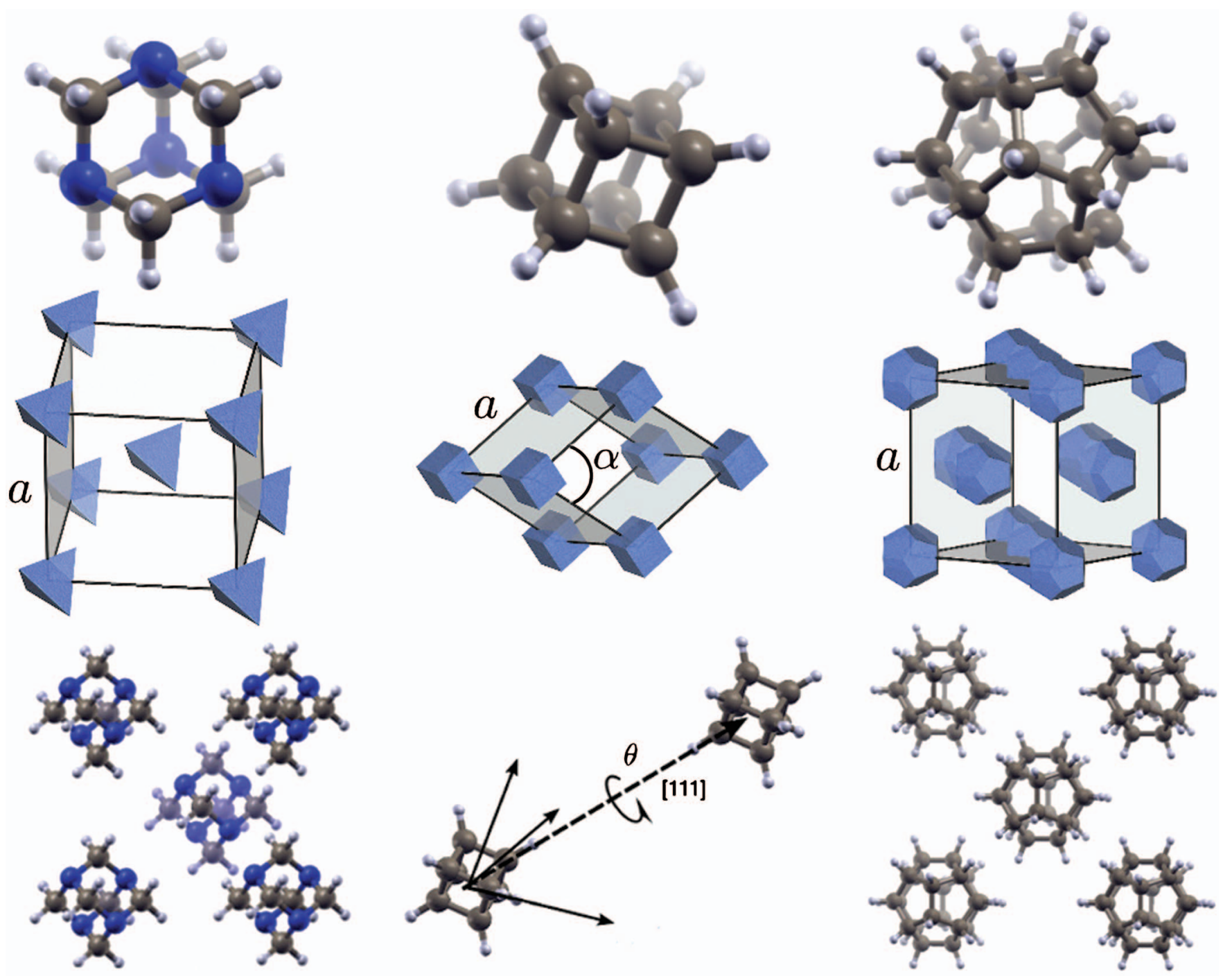

FIG. 1. The molecular and crystal structure of hexamine, dodecahedrane, and cubane. The upper panels show the respective molecular structures. The midpanels give schematics of the crystal structures, where the molecular symmetry and orientation are highlighted by the use of a tetrahedron, dodecahedron, and a cube in place of the molecules. The lower panel shows, for hexamine and dodecahedrane, facets of the crystal structure and, for cubane, the orientation of the cubane molecule in the unit cell. Hexamine has a bcc unit cell, dodecahedron has an fcc unit cell, and cubane has a rhombohedral unit cell, with equal angles $\alpha$ between the lattice vectors.

sents the molecules hexamine, cubane, and dodecahedrane and their experimental crystal structures. The third section deals with the computational details of vdW-DF. The fourth gives the results for lattice parameters and bulk modulus. In the fifth section, we compare the asymptotic $1 / r^{6}$-form of van der Waals interactions with the full nonlocal correlation for different molecules and the vdW-DF potential energy curve with DFT-D calculations for a cubane dimer. The final section holds our conclusions.

\section{PLATONIC MATTER}

Plato asserted that the basic building blocks of Nature were five geometrical structures, today known as platonic solids. ${ }^{32}$ These are the five convex polyhedra with all faces, edges, and angles congruent. On the molecular level, two of these solids have synthetic hydrocarbon (compounds of only carbon and hydrogen) analogs: cubane $\left(\mathrm{C}_{6} \mathrm{H}_{6}\right)$, which corresponds to the cube, and dodecahedrane $\left(\mathrm{C}_{20} \mathrm{H}_{20}\right)$, which corresponds to the 12-faced dodecahedron. The fascination mathematicians held for these geometrical structures since antiquity echoed in the more recent struggle to synthesize their hydrocarbon representatives. The highly strained bonds posed the main challenge. Obstacles were eventually overcome and in 1964 cubane was synthesized ${ }^{33}$ followed by dodecahedrane in $1978 .^{34} \mathrm{~A}$ third, tetrahedrane exists only within a larger chemical structure ${ }^{35}$ and no crystallographic characterization exists. Tetrahedrane does therefore not represent a good testing ground for vdW-DF, and we instead study the molecular crystal of hexamine $\left(\mathrm{C}_{6} \mathrm{H}_{12} \mathrm{~N}_{4}\right)$. Although not a hydrocarbon, it shares several features with the platonic hydrocarbons: it is organic, its nonhydrogen atoms form a cage, and this cage has the symmetry of platonic solid tetrahedron. The top row in Fig. 1 shows the molecular structures (from left to right) of hexamine, cubane, and dodecahedrane ordered according to the ascendancy of their platonic analogs.

The second and third rows in Fig. 1 show the crystal structures. The first column shows hexamine, the middle 
shows, cubane, and the final column, dodecahedrane. The crystal structure of hexamine forms a bcc with a single molecule in each unit cell and $\bar{I} \overline{4} 3 \mathrm{~m}$ symmetry. Hexamine has been used as a model system in numerous studies, ${ }^{36-38}$ and the crystal structure was determined as early as in $1923 .^{39}$ The crystal structure of cubane is rhombohedral with equal external angles and a single molecule in each cell. The molecule is oriented according to the $\mathrm{R} \overline{3}$ space group; a rotation about the [111] axis specifies the configuration of the molecule. ${ }^{31}$ The crystal structure of dodecahedrane is fcc, also with a single molecule in each cell and $\mathrm{Fm} \overline{3}$-space group. $^{40}$

Hexamine finds numerous industrial uses. ${ }^{41}$ For instance, it serves as a component in fuel tablets and as an antibiotic. ${ }^{42}$ The platonic hydrocarbons find only hypothetical applications; cubane has been identified as a potential high-energy fuel and explosive. ${ }^{43}$

In addition to being well-studied crystals, especially hexamine and cubane, these crystals make attractive testing grounds for vdW-DF for two more reasons. First, their simple structures allow for brute-force determination of lattice parameters. Within the crystal symmetry, this determination corresponds to mapping out the potential energy of a single parameter for hexamine and dodecahedrane and three for cubane. The brute-force approach facilitates postprocessing analysis, such as computation of bulk modulus, ${ }^{44}$ and makes it easier to evaluate how the choice of exchange in DFT affects crystal structure and cohesion energy. Second, the high symmetry of the molecules reduces the large set of atom-to-atom $C_{6}$ coefficients to only a few equivalent values. This reduction simplifies the comparison of full vdW-DF calculations with atom-based asymptotic accounts of the van der Waals forces.

\section{COMPUTATIONAL DETAILS}

The crystal structure and bulk modulus of hexamine, cubane, and dodecahedrane are determined with DFT using a nonlocal-density functional called vdW-DF. The traditional semilocal GGA for exchange-correlation provides accurate bond lengths and charge-density $n(\mathbf{r})$ but fails to capture correlated motion of separated electrons: the long-range dispersion forces. vdW-DF includes these correlations and can therefore account for the structure and cohesion of sparse matter. Since details of the functional and its implementation are given elsewhere, ${ }^{10,21,45-47}$ we focus mostly on computational steps specific for determination of crystal structures.

The nonlocal correlation of vdW-DF takes the form of a double-space integral,

$$
E_{c}^{\mathrm{n} l}[n]=\frac{1}{2} \int_{V_{0}} d \mathbf{r} \int_{V} d \mathbf{r}^{\prime} n(\mathbf{r}) \phi\left(\mathbf{r}, \mathbf{r}^{\prime}\right) n\left(\mathbf{r}^{\prime}\right),
$$

over an interaction kernel $\phi\left(\mathbf{r}, \mathbf{r}^{\prime}\right)$. Here $V_{0}$ denotes the central unit cell and $V$ (formally) the entire space. The kernel can be tabulated in terms of two parameters $d$ and $d^{\prime}$ related to the local response $q_{0}(\mathbf{r})$ and spatial separation $\left|\mathbf{r}-\mathbf{r}^{\prime}\right|$ by $d=q_{0}(\mathbf{r})\left|\mathbf{r}-\mathbf{r}^{\prime}\right|$ and $d^{\prime}=q_{0}\left(\mathbf{r}^{\prime}\right)\left|\mathbf{r}-\mathbf{r}^{\prime}\right|$. The remaining part of the exchange-correlation functional of vdW-DF consists of the exchange part of revPBE (Ref. 48) and the correlation of LDA

$$
E_{\mathrm{xc}}^{\mathrm{vdWDF}}=E_{c}^{\mathrm{LDA}}+E_{x}^{\mathrm{revPBE}}+E_{c}^{\mathrm{nl}}[n] .
$$

The total energy functional of vdW-DF, $E^{\mathrm{vdWDF}}[n]$, also includes the standard elecrostatic and kinetic-energy terms within the Kohn-Sham scheme. ${ }^{49}$ It is convenient to write this energy in terms of a semilocal part $E_{0}[n]$ containing all but the nonlocal correlation so that $E^{\mathrm{vdWDF}}[n]=E_{0}[n]$ $+E_{c}^{\mathrm{nl}}[n]$. For input charge-density $n(\mathbf{r})$, we use the result of semilocal calculations with the PBE (Ref. 5) flavor of GGA. We will refer to calculations with the PBE flavor of GGA as DFT-GGA. The charge density could also have been obtained within fully self-consistent vdW-DF. ${ }^{45,50}$ However, the two-step non-self-consistent procedure introduces only a slight approximation. Previous studies have documented that for systems with small charge transfer, binding energies of non-self-consistent vdW-DF only differ by tiny amounts from fully self-consistent energies. ${ }^{45,51}$

To speed up evaluation of the nonlocal correlation, we introduce a radius cutoff based on the decay of van der Waals forces at large separations. With this cutoff, the kernel takes form

$$
E_{c}^{\mathrm{n} \mathrm{l}}[n] \approx \frac{1}{2} \int_{V_{0}} d \mathbf{r} n(\mathbf{r}) \int_{\left|\mathbf{r}-\mathbf{r}^{\prime}\right|<R} d \mathbf{r}^{\prime} \phi\left(\mathbf{r}, \mathbf{r}^{\prime}\right) n\left(\mathbf{r}^{\prime}\right) .
$$

In the above expression, we see that the CPU-cost for evaluating the nonlocal part of vdW-DF goes as $R^{3} \mathcal{O}\left(V_{0}\right)$. Thus, for large or periodic systems, the computational costs increase linearly with system size. To cut computational costs further, we introduce an extra radius cutoff corresponding to the separation between dense and sparse sampling of the charge-density grid. We note that Ref. 50 reports a more elaborate scheme which considerably reduces CPU-costs, yet our simple measure was sufficient for our non-self-consistent calculations as the underlying DFT-GGA calculations of electronic density dominated time consumption.

The use of revPBE for exchange in vdW-DF was motivated by the fact that this exchange functional excludes unphysical binding effects at large distances. ${ }^{15,46}$ For a range of systems, vdW-DF overestimates binding separations. ${ }^{21,46}$ Several studies indicate that this discrepancy can be attributed to the details of the exchange functional. ${ }^{21,51-54}$ Puzder et $a l^{52}$ demonstrated that replacing Hartree-Fock exchange with revPBE improves binding separations for benzene dimers. Gulans et $a l^{53}$ showed that for a selected range of molecular complexes the PBE exchange functional improves binding energies. We furthermore illustrate the sensitivity to exchange by including results based on use of an alternative $v d W-D F(P B E)$, where revPBE exchange has been replaced by that of PBE. We do not argue for replacing vdW-DF with vdW-DF(PBE); instead we simply explore consequences of a different account of exchange. ${ }^{54}$

To calculate crystal parameters and cohesion energy, we minimize the potential energy. In many respects this vdW-DF structure determination is similar to those in Refs. $2,23,47$, and 55 . The potential energy is given by the dif- 
ference between the total energy of the full crystal and a reference energy for a system of isolated molecules

$$
E_{\mathrm{coh}}(a,\{\alpha \theta\})=E^{\mathrm{vdWDF}}(a,\{\alpha \theta\})-E^{\mathrm{vdWDF}}(a \rightarrow \infty,\{\alpha \theta\}) .
$$

In the above equation, the curly brackets are specific for the cubane crystal as it depends on three rather than one parameter. In the reference calculation corresponding to the reference energy, $E^{\mathrm{vdWDF}}(a \rightarrow \infty,\{\alpha \theta\})$, the semilocal part is obtained somewhat differently from the part containing the nonlocal correlation. For the former, we effectively isolate the molecules in our periodic boundary calculations by using a unit cell of doubled size in all directions. This measure secures negligible charge overlap between the molecules in the supercells. For the latter (nonlocal) part, we restrict the integral of Eq. (3) to the central supercell to avoid coupling between the enlarged unit cells. Hence, only nonlocal correlations within the molecule contribute to the reference energy.

To enhance accuracy in the evaluation of the nonlocal part of the potential energy, we systematically cancel a small but noticeable grid dependence in the evaluation of Eq. (3). This cancellation is performed by making sure to use the same fast Fourier transform (FFT) grid spacing in the reference calculation as in the main calculation. Furthermore, we make sure to place the isolated molecules in the same relative configuration to the underlying grid in the reference calculation as in the main calculation. We thus perform an additional reference calculation for every molecular configuration investigated. These measures have been used to secure a high accuracy of the nonlocal part of vdW-DF in several earlier studies. $2,46,47,55$

We map the potential energy landscape by varying the lattice parameters of the molecular crystals within the experimental crystal symmetry. The stiff cage molecules allow us to keep the internal coordinates of the molecules frozen for all configurations. The molecular structures are determined in isolation using the PBE flavor of GGA. The resulting bond lengths will be compared with experimental data in the next section to verify the utility of conventional DFT-GGA for the internal structure of strained molecules.

The electronic structure calculations rely on the planewave code DACAPO (Ref. 56) using ultrasoft pseudopotentials. In combination with a separate reference calculation as previously discussed, we secure the convergence of the nonlocal correlation by specifying an FFT-grid spacing less than $0.13 \AA$. This spacing leads to an effective plane-wave energy cutoff of at least $500 \mathrm{eV}$. For all crystals, we set the Monkhorst-Pack k-sampling to $4 \times 4 \times 4$.

\section{STRUCTURE DETERMINATION}

\section{A. Molecular structure}

Table I shows the calculated molecular structures. It demonstrates DFT-GGA can account for the intramolecular bonding even in the highly strained cubane molecules. The resulting bond lengths differ by less than $1 \%$ from the ex-
TABLE I. Experimental and calculated bond lengths of hexamine, cubane, and dodecahedrane. The calculations were done with the PBE flavor of GGA. $l$ denotes the $\mathrm{C}-\mathrm{C}$ bond length for cubane and dodecahedrane and the $\mathrm{C}-\mathrm{N}$ bond length for hexamine. $l_{\mathrm{CH}}$ denotes the carbon-hydrogen bond length.

\begin{tabular}{lccc}
\hline \hline Parameter & Hexamine & Cubane & Dodecahedrane \\
\hline$l(\AA)$ & 1.472 & 1.566 & 1.549 \\
$l^{\text {expt }}(\AA)$ & $1.476^{\mathrm{a}}$ & $1.562^{\mathrm{b}}$ & $1.544^{\mathrm{c}}$ \\
$l_{\mathrm{CH}}(\AA)$ & 1.101 & 1.095 & 1.100 \\
$l_{\mathrm{CH}}^{\text {ext }}(\AA)$ & $1.088^{\mathrm{a}}$ & $1.097^{\mathrm{b}}$ & $\ldots$ \\
\hline
\end{tabular}

${ }^{\mathrm{a}}$ Reference 57.

${ }^{\mathrm{b}}$ Reference 58.

${ }^{\mathrm{c}}$ Reference 59.

perimental values. In first-principle studies of molecular crystals, accurate determination of lattice parameters requires accurate account of constituent molecules.

Unlike many calculations, where determination of crystal structure often starts from the structure of the individual molecules, most experiments resolve the molecular structure by looking at the diffraction pattern of a full molecular crystal. For our purposes of testing the first-principle vdW-DF method, this is fortunate, as efforts to characterize molecular structures also generate an abundance of experimental data on molecular crystal structures.

\section{B. Crystal structure}

Figure 2 shows the binding curves and contours found by varying the molecular crystals' lattice parameters identified in Fig. 1. The simple crystal structures of hexamine and dodecahedrane give a one-dimensional potential energy landscape, while the cubane crystal has a three-dimensional one. In the right panel of Fig. 2, we display the $\alpha a$ and the $\alpha \theta$ intersections, for the optimal values of $\theta$ and $a$. The curves exhibit a pronounced asymmetry around their minimum. This asymmetry arises because the van der Waals attraction is much softer than the kinetic energy repulsion.

Table II contains the calculated results and experiment values. Standard vdW-DF performs well both for lattice parameters and cohesion energy. If not directly available, we obtain experimental cohesion energies by correcting for gas phase and vibrational contributions to the enthalpy of sublimation, using the method described in Refs. 64 and 65.

The bulk modulus is obtained with use of polynomial interpolation according to the scheme of Ziambaras and Schröder. ${ }^{44}$ The required polynomial were constructed using data from selected one-dimensional deformations. ${ }^{66}$ For hexamine, where the experimental bulk modulus is available, the computed value show fair agreement with the experimental value. There is also a trend for bigger cage molecules to have a larger bulk modulus. We attribute this trend to the fact that for bigger molecules a smaller relative part of the unit cell consists of soft intramolecular regions. Therefore, as the relative unit-cell dimensions change, the distance between (stiff) molecules changes more for big molecules than for small molecules. 

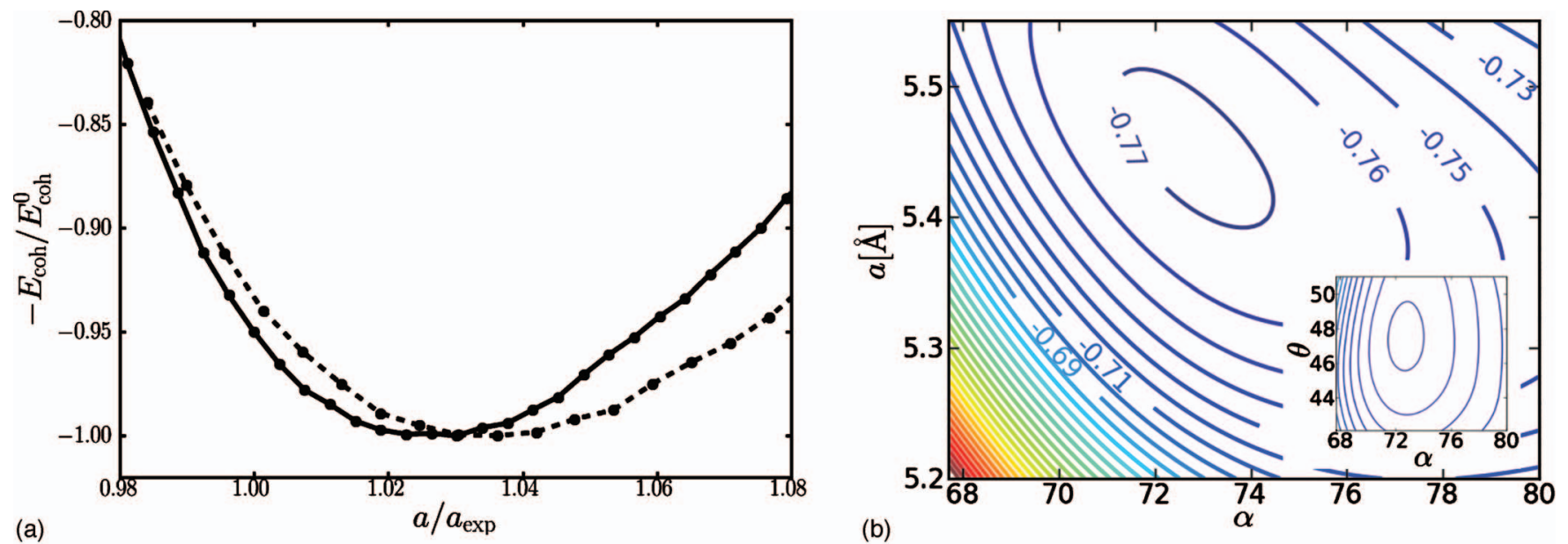

FIG. 2. The potential energy curves for the crystal of hexamine and dodecahedrane (left panel) and corresponding contour plots for the crystal of cubane (right panel). The left panel shows potential energy where the curves are normalized separately so that the experimental lattice parameter and the calculated cohesion energy equal unity. The solid curve represents dodecahedrane and the dashed hexamine. The difference in the optimal $a / a_{\text {expt }}$ value can be attributed to the experimental lattice parameter being measured at low temperature for hexamine but not for dodecahedrane. The right panel shows spline-interpolated contour plots of the two-dimensional intersection of the three-dimensional potential energy landscape. The main figure corresponds to the optimal value of the internal angle $\theta$, while the insert corresponds to the optimal value of the unit-cell length $a$. The pronounced asymmetry of the curves and contours reflect the hard wall provided by Pauli repulsion.

For all three crystals, we find unit-cell volumes somewhat larger than the experimental ones. Similar overestimations have also been encountered in previous studies. ${ }^{21,46,54}$ The alternative choice of PBE as exchange functional, vdW$\mathrm{DF}(\mathrm{PBE})$, influences results substantially. On one hand, it improves lattice parameters almost to the level of standard DFT for intramolecular bonds. On the other hand, the value of cohesive energy and bulk modulus worsens. These results signal that the main discrepancy between experiments and vdW-DF stems from the specific form of semilocal exchange. ${ }^{21,52-54}$

TABLE II. The vdW-DF prediction of lattice parameters, cohesion energy, and bulk modulus for the crystals of hexamine, cubane, and dodecahedrane compared with an alternative vdW-DF(PBE) based on PBE exchange and with experimental values. The experimental lattice parameters are based on low temperature measurements, except for dodecahedrane.

\begin{tabular}{lcccc}
\hline \hline & Parameter & vdW-DF & vdW-DF (PBE) & Expt. \\
\hline Hex. & & & & \\
& $a(\AA)$ & 7.14 & 6.93 & $6.910^{\mathrm{a}}$ \\
& $E_{\text {coh }}(\mathrm{eV})$ & -1.0127 & -1.427 & $-0.827^{\mathrm{b}}$ \\
& $B_{0}(\mathrm{GPa})$ & 10.0 & 14.0 & $7.0^{\mathrm{c}}$ \\
Cub. & & & & \\
& $a(\AA)$ & 5.45 & 5.25 & $5.20^{\mathrm{d}}$ \\
& $\alpha$ & 73 & 72.5 & $72.7^{\mathrm{d}}$ \\
& $\theta$ & 47.5 & 46.5 & $46^{\mathrm{d}}$ \\
& $E_{\text {coh }}(\mathrm{eV})$ & -0.77 & -1.15 & $-0.857^{\mathrm{e}}$ \\
Dod. & $B_{0}(\mathrm{GPa})$ & 7.2 & 14.8 & $\cdots$ \\
& $a(\AA)$ & 10.92 & 10.56 & $10.60^{\mathrm{f}}$ \\
& $E_{\text {coh }}(\mathrm{eV})$ & -1.46 & -2.06 & $\ldots$ \\
& $B_{0}(\mathrm{GPa})$ & 12.2 & 18.6 & $\cdots$ \\
\hline \hline
\end{tabular}

\footnotetext{
${ }^{2}$ Reference 57.

${ }^{\mathrm{b}}$ Reference 60.

${ }^{\mathrm{c}}$ Reference 61.

${ }^{\mathrm{d}}$ Reference 57.

${ }^{\mathrm{e}}$ Reference 62 .

${ }^{\mathrm{f}}$ Reference 63.
}

We note that an ab initio study of cubane has previously been performed at the LDA level. ${ }^{67}$ Being a local functional, LDA has no physical basis for the van der Waals binding that provides the cohesion of this molecular crystal. The spurious LDA binding arises from an unphysical accounts of exchange. ${ }^{15,46}$ Once the molecular crystals are investigated with DFT-GGA, which has an improved account of exchange, the binding essentially vanishes. ${ }^{67}$

\section{ASYMPTOTIC PAIR POTENTIALS VERSUS NONLOCAL CORRELATION}

The asymptotic van der Waals interactions between two atoms or molecules goes as the simple power law $C_{6} / r^{6}$, where $C_{6}$ gives the strength of the interaction. This familiar result can be derived from second-order perturbation theory ${ }^{68}$ or from an analysis of the shifts in the zero-point motions of the electron. ${ }^{69} \mathrm{~A}$ common strategy in force-field methods and empirical extensions of DFT is to adopt such an asymptotic form at all separations in terms of atom-centered pair potentials (APP). However, we cannot take for granted that the asymptotic behavior should hold for separations closer to that of intramolecular binding. On the contrary, because van der Waals forces arise from correlated motion of electrons and not from the atomic nuclei, several mechanisms affect and enhance the interaction at short and intermediate separations: higher order moments contribute, polarizability changes as charge is distorted, and finite-k dispersion of the electronic response becomes important. Zaremba and Kohn ${ }^{70}$ considered adsorption of noble atoms on surfaces and documented a significant enhancement of dispersion energies over an atom-centered account; their asymptotic $1 / d^{3}$ form uses the distance to an image plane $d$ rather than to the surface atoms. Within vdW-DF, Kleis et $a l .^{23}$ demonstrated that for interactions in nanotube bundles, the force stems primarily from the electron tail around the nanotube and that as the tubes get closer, higher order mo- 
ments dominate over the asymptotic interaction. Part of this enhancement can be interpreted as an image-plane effect.

Here we investigate whether an atom-centered $1 / r^{6}$ form is a good approximation for the nonlocal correlation of molecular dimers. As our argument is based on the asymptotic vdW-DF account of van der Waals forces, we also discuss for these molecules the accuracy of the asymptotic account. In the first subsection, we compare the nonlocal correlation of vdW-DF for dimers of hexamine, cubane, and dodecahedrane with its corresponding APPs. We document a significant enhancement of the nonlocal correlation at short (binding) separations and at intermediate separations, one to three Ångström beyond typical binding separations. In the second subsection, we discuss the accuracy of our $C_{6}$ coefficients. In the third, we investigate consequences of this result, in particular for the use of DFT-D. We will argue that although standard DFT-D methods can provide good descriptions of both short and asymptotic separations, their asymptotic atom-centered form does not describe the enhancement of correlation at intermediate separations exhibited by vdW-DF.

\section{A. Asymptotic vdW-DF}

For the asymptotic van der Waals forces, the $C_{6}$ coefficient between two fragments, $A$ and $B$, can be computed from the general formula

$$
C_{6}^{A B}=\frac{3}{\pi} \int_{0}^{\infty} d u \alpha_{A}(i u) \alpha_{B}(i u),
$$

where $\alpha(\omega)$ is the polarizability of the fragment. In order to calculate the $C_{6}$ coefficients, we approximate $\alpha(\omega)$ with the local external-field susceptibility of vdW-DF,

$$
\chi_{A}^{\mathrm{vdW}-\mathrm{DF}}(\omega, \mathbf{r})=\frac{n_{A}(\mathbf{r})}{\left[9 q_{0}(\mathbf{r})^{2} / 8 \pi\right]^{2}-\omega^{2}},
$$

and a polarizability given by

$$
\alpha_{A}^{\mathrm{vdW}-\mathrm{DF}}(\omega)=\int d^{3} \mathbf{r} \chi_{A}^{\mathrm{vdW}-\mathrm{DF}}(\omega, \mathbf{r}) .
$$

To generate vdW-DF based APPs (APP-vdWDF), we first partition the full charge-density $n(\mathbf{r})=\sum_{i} n_{i}(\mathbf{r})$ among the atoms of the molecules with aid of Bader analysis. ${ }^{71,72}$ Based on this charge partition, we calculate the atom-to-atom $C_{6}$ coefficients using Eq. (5). Initially, this procedure generates $N^{2}$ different $C_{6}$ coefficients for a molecular dimer of $N$ atoms per molecule. For a dodecahedrane dimer, we get as much as $40 \times 40=1600$ coefficients. Fortunately, because of the high symmetry of the isolated molecules, this number reduces to only three equivalent values for cubane and dodecahedrane: $C_{6}^{\mathrm{C}-\mathrm{C}}, C_{6}^{\mathrm{C}-\mathrm{H}}$, and $C_{6}^{\mathrm{H}-\mathrm{H}}$. For hexamine, the extra nitrogen atoms lead to six coefficients. As noise in the electronic density affects the value of the coefficients, we average over a large set of equivalent values to obtain the final values.

The upper part of Table III shows the $C_{6}$ coefficients calculated within asymptotic vdW-DF, both as partitioned according to the Bader analysis and as evaluated for the entire molecule. The calculated coefficients per atomic pair deviate much from a naive assignment of the full coefficient of the molecule according to the number of valence electrons of
TABLE III. Computed values of the $C_{6}$ coefficients (Hartree atomic units) for different pairs of atoms within respective molecules using the asympotic form of vdW-DF with charge density as partitioned with a Bader analysis. The figure also shows the molecule-molecule $C_{6}$ coefficients obtained with the ALL scheme (Ref. 73) and the molecule-molecule $C_{6}$ coefficents obtained with the use of parameters given in DFT-D schemes (Refs. 16-18).

\begin{tabular}{lccc}
\hline \hline$C_{6}^{\text {vdWDF }}$ & Hex & Cub & Dod \\
\hline $\mathrm{C}-\mathrm{C}$ & 4.44 & 13.5 & 11.2 \\
$\mathrm{C}-\mathrm{H}$ & 4.04 & 6.87 & 6.45 \\
$\mathrm{H}-\mathrm{H}$ & 3.98 & 3.72 & 4.07 \\
$\mathrm{~N}-\mathrm{N}$ & 32.6 & $\ldots$ & $\ldots$ \\
$\mathrm{N}-\mathrm{C}$ & 12.0 & $\ldots$ & $\ldots$ \\
$\mathrm{N}-\mathrm{H}$ & 11.0 & $\ldots$ & $\ldots$ \\
mol-mol (vdW-DF) & 3470 & 1990 & 11300 \\
mol-mol (ALL) & 3270 & 1940 & 10200 \\
mol-mol (Wu) & 4340 & 2600 & 16300 \\
mol-mol (Grimme) & 4000 & 2630 & 16400 \\
mol-mol (Jurečka) & 4790 & 3120 & 19500 \\
\hline \hline
\end{tabular}

the underlying atom. In such a scheme, the $\mathrm{C}-\mathrm{C}$ coefficient would be 16 times larger than the $\mathrm{H}-\mathrm{H}$ coefficient, while in fact, for cubane and dodecahedrane, it is merely three to four times stronger. This result can be attributed to the relative stronger response of the low-density regions surrounding the hydrogen atoms as $q_{0}(\mathbf{r}) \propto[n(\mathbf{r})]^{1 / 3}$ (in the homogeneous limit), and these areas dominate $E_{c}^{\mathrm{nl}}$. The somewhat anomalous values for hexamine can be attributed to our Bader analysis scheme partitioning a significant portion of the charge density near the carbon atoms to the centrally located nitrogen atoms. ${ }^{74}$ For cubane and dodecahedrane, the partitioning was similar, and the differences in atomistic $C_{6}$ values show that they are influenced by their local environment.

Having generating $C_{6}$ coefficients appropriate for a comparison between the asymptotic account and the full correlation of vdW-DF, we study dimers of hexamine, cubane and dodecahedrane at different separations. We choose orientations that are given by the nearest-neighbor configurations in the crystals. The total asymptotic nonlocal correlation of APP-vdWDF reads

$$
E_{\mathrm{app}}^{\mathrm{vdWDF}}=\sum_{i} \sum_{j} \frac{C_{6}^{i j}}{\left|\mathbf{r}_{i}-\mathbf{r}_{j}^{\prime}\right|},
$$

where $i$ and $j$ label atoms in separate molecules of the dimer.

Figure 3 shows results for the full nonlocal correlation of vdW-DF (solid curve) and that of APP-vdWDF (dashed curve). At large separations these two curves converge. In contrast, they differ significantly at relevant binding (short) separations and at intermediate separations. At these separations, the nonlocal correlation is almost double as large as that of APP-vdWDF, for both hexamine and cubane, while for the biggest molecule, dodecahedrane, the difference is smaller. For all dimers, we also need to go to relatively large separations to recover asymptotic values for the nonlocal correlation.

As vdW-DF is an $a b$ initio functional, based on a set of exact sum rules, ${ }^{10}$ the strong enhancement of nonlocal correlations at short and intermediate separations indicates that the asymptotic form neglects important contributions. It also 


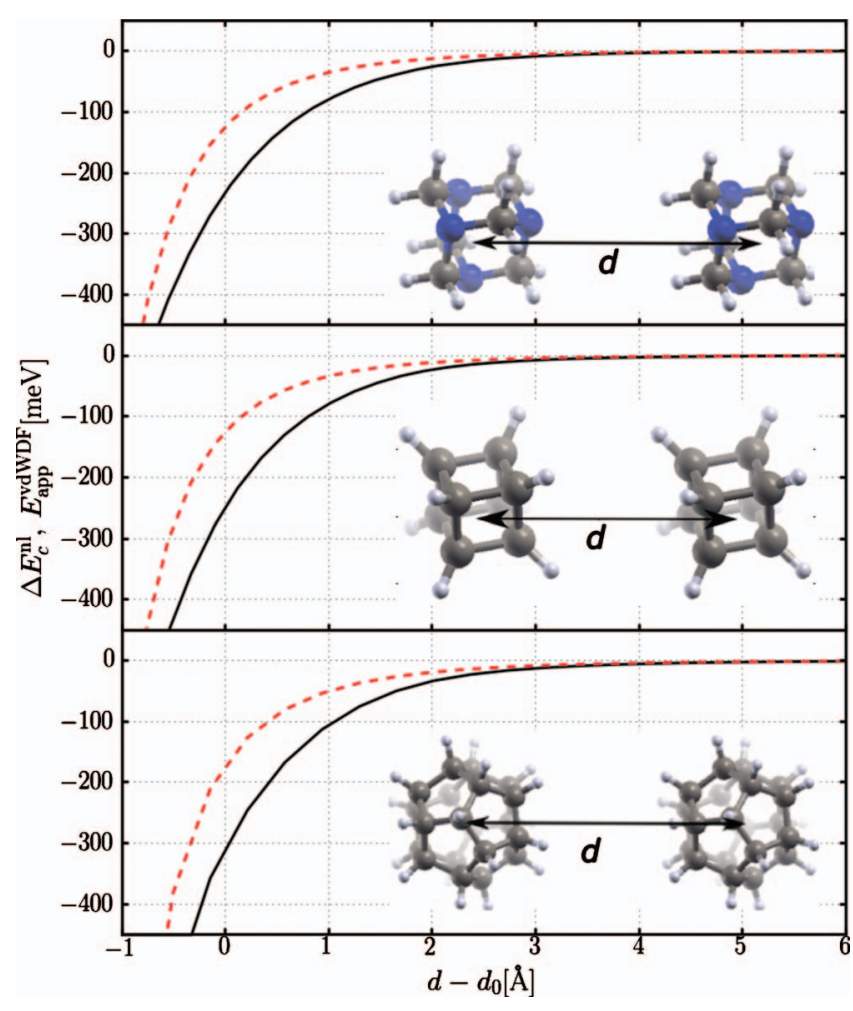

FIG. 3. Comparison between the nonlocal correlation of vdW-DF and atomic pairs potentials generated with asymptotic vdW-DF for a dimer of hexamine, dodecahedrane, and cubane (from top to bottom) in configurations corresponding to nearest neighbors in their respective crystal. The dashed curve gives the APP-vdWDF result, while the solid curve gives nonlocal correlation of vdW-DF: $\Delta E_{\mathrm{c}}^{\mathrm{nl}}(d)=E_{\mathrm{c}}^{\mathrm{nl}}(d)-E_{\mathrm{c}}^{\mathrm{nl}}(d \rightarrow \infty)$. The horizontal axis gives the difference from the vdW-DF crystal binding separation $d_{0}$, which respectively takes the values $6.18,5.45$, and $7.72 \AA$. The difference between the two curves demonstrates the enhancement of nonlocal correlations over the vdW-DF asymptotic atom-based account at relevant binding separations and intermediate separations.

highlights that in constructing modified semiempirical van der Waals functionals, ${ }^{11,12}$ a fitting of the asymptotic functional to $C_{6}$ coefficients does not guarantee an accurate description at short binding separations.

\section{B. Comparison of $C_{6}$ coefficients}

The lower part of Table III, gives the molecular $C_{6}$ coefficients calculated with asymptotic vdW-DF, the Andersson-Langreth-Lundqvist (ALL) scheme, ${ }^{73}$ and computed using the atomic coefficients of Wu et al., ${ }^{16} \mathrm{Grimme},{ }^{17}$ and Jurečka et al. ${ }^{18}$ The ALL and vdW-DF give coefficients smaller than that used in DFT-D schemes.

We expect that Grimme and Wu provide good values for molecular $C_{6}$ coefficients, because their underlying atomistic coefficients were fitted to reproduce a range of accurate molecular $C_{6}$ coefficients calculated from experimental molecular polarizabilities (Ref. 16 and references therein). The coefficients of Jurečka ${ }^{18}$ give somewhat larger molecular $C_{6}$ coefficients. This comes from the use of Slater-Kirkwood average $^{75}$ for $C_{6}$ coefficients between different atomic species while keeping those of Grimme for identical atomic species (the $C_{6}$ coefficients of Grimme are optimized for a different average).
TABLE IV. Atomistic $C_{6}$ coefficients for cubane used in APP-vdWDF (calculated with asymptotic vdW-DF and charge density partitioned with Bader analysis) and coefficients used in DFT-D methods.

\begin{tabular}{lrrcc}
\hline \hline$C_{6}^{\text {vdWDF }}$ & vdW-DF & Wu $^{\mathrm{a}}$ & Grimme $^{\mathrm{b}}$ & Jurečka $^{\mathrm{c}}$ \\
\hline $\mathrm{C}-\mathrm{C}$ & 13.5 & 22.06 & 28.3 & 28.3 \\
$\mathrm{C}-\mathrm{H}$ & 6.8 & 7.89 & 5.01 & 8.82 \\
$\mathrm{H}-\mathrm{H}$ & 3.7 & 2.83 & 2.75 & 2.75 \\
\hline \hline
\end{tabular}

${ }^{\mathrm{a}}$ Reference 16.

${ }^{\mathrm{b}}$ Reference 17 .

${ }^{\mathrm{c}}$ Reference 18 .

Asymptotic vdW-DF and ALL likely underestimates the $C_{6}$ coefficients for these molecules; they are $20 \%-30 \%$ smaller than that used in DFT-D methods. For the similar ALL scheme, Ref. 76 reports an underestimation of $C_{6}$ coefficients for larger molecules, in particular for benzene and C60. A difference between the ALL scheme and asymptotic $\mathrm{vdW}-\mathrm{DF}$ is that for the former a hard cutoff accounts for plasmon damping, while for the latter, the local response $q_{0}(\mathbf{r})$ provides a smooth cutoff. There is good consistency between the two methods. Both methods also assume a local, scalar relationship between the applied and the full electric field, which is an approximation for finite-sized objects. ${ }^{73} \mathrm{We}$ speculate that this approximation contributes to the underestimation of $C_{6}$ coefficient for the investigated relatively large molecules.

Table IV shows atomistic $C_{6}$ coefficients for cubane as calculated with asymptotic vdW-DF and given by $\mathrm{Wu},{ }^{16}$ Grimme, $^{17}$ and Jurečka ${ }^{18}$ for use in DFT-D schemes. vdW-DF weighs the relative response of the carbon less than that of the hydrogen, compared with the values of DFT-D. ${ }^{74}$ This property could relate to the above-mentioned approximate treatment of electrodynamics. It could also relate to the carbon atoms being located somewhat inside the molecule, having a different local charge density and responding less to external fields than an atom on the exterior would; in contrast, DFT-D does not discriminate between atoms at different locations.

\section{Consequences for atom-based pair potentials: The binding curve of cubane}

The vdW-DF results presented in the first subsection show that a simple asymptotic account only partially captures correlation effects at short and intermediate separations. As DFT-D uses such an asymptotic form to describe nonlocal correlations, this result stands in apparent contrast to the many successful applications of DFT-D. ${ }^{16-18,77-79}$

To understand consequences of our result for methods such as DFT-D, we must first consider other effects that could contribute to the difference between APP-vdWDF and the nonlocal correlation of vdW-DF. In vdW-DF, correlations are described by $E_{c}^{\mathrm{LDA}}+E_{c}^{\mathrm{nl}}$, and hence $E_{c}^{\mathrm{nl}}$ also accounts for semilocal correlations. ${ }^{10,46}$ Second, we must consider the specific designs of actual DFT-D schemes because these could counteract the lack of enhancement of nonlocal correlations. To describe exchange-correlation, DFT-D combines 


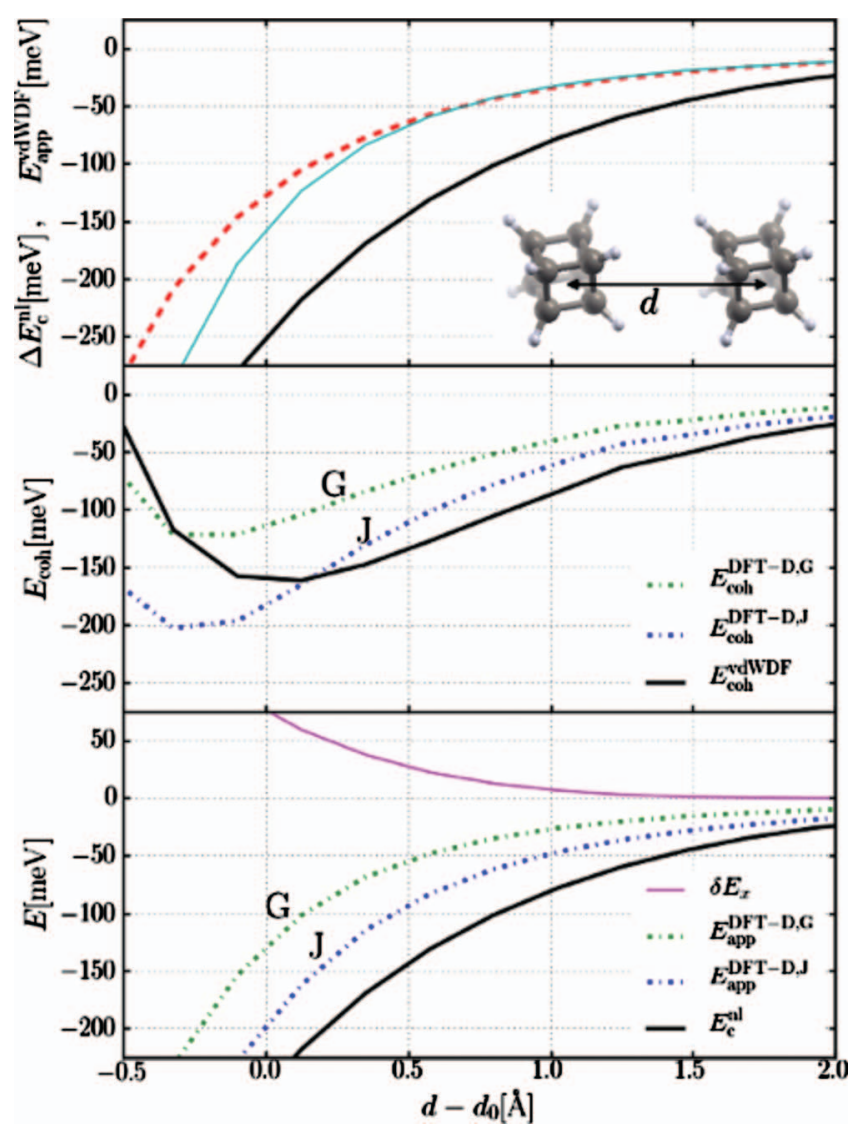

FIG. 4. Comparison between different accounts of nonlocal correlation for a dimer of cubane. In the upper panel, the dashed curve gives APP-vdWDF. The thin solid curve gives the sum of APP-vdWDF and the correlation of PBE. The thick solid curve gives the nonlocal correlation of vdW-DF. In the middle panel, the thick curve gives the cohesion energy using vdW-DF, $E_{\mathrm{coh}}^{\mathrm{vdWDF}}(d)$; the upper (lower) dash-dotted curve gives the DFT-D binding curve as given by Grimme, $E_{\mathrm{coh}}^{\mathrm{DFT}-\mathrm{D}, \mathrm{G}}(d)$ (Jurečka, $E_{\mathrm{coh}}^{\mathrm{DFT}-\mathrm{D}, \mathrm{J}}(d)$. The lower panel gives the corresponding nonlocal correlation of vdW-DF and Grimme (Jurečka). The thin solid curve gives the difference between exchange of revPBE and PBE. The horizontal axis gives the difference from the vdW-DF crystal binding separation $d_{0}$ for cubane.

the asymptotic atom-centered form with a semilocal GGA account. It also introduces fitting parameters to be used in combination with a specific GGA flavors.

The top panel of Fig. 4 shows that gradient corrections do not account for the difference between the full vdW-DF and the APP-vdWDF results. For APP-vdWDF, we can combine the purely semilocal correlation of PBE with APPvdWDF (in a new description APP-mod),

$$
E_{\mathrm{APP}-\bmod }^{\mathrm{vdWDF}}(d)=E_{\mathrm{APP}}^{\mathrm{vdWDF}}(d)+\Delta E_{c}^{\mathrm{PBE}}(d)-\Delta E_{c}^{\mathrm{LDA}}(d),
$$

to assess the magnitude of purely semilocal corrections relative to the difference between vdW-DF and APP-vdWDF. In this APP account, we have introduced the LDA and PBE terms: $\Delta E_{c}(d)=E_{c}(d)-E_{c}(d \rightarrow \infty)$.

We focus our discussion on the cubane dimer. The lower thin solid curve gives APP-mod; the thick solid curve gives the nonlocal correlation of vdW-DF. The curves are shown as a function of the intermolecular distance $d$, with $d_{0}$ indicating the vdW-DF binding distance in the crystal. At short separations, the thin curve lies closer to the thick curve than the corresponding APP-vdWDF result (thick dashed curve).
Thus, some of the difference between APP-vdWDF and the nonlocal correlation arise from a lack of semilocal correlation contributions in APP-vdWDF. ${ }^{10}$ However, even with this inclusion the difference is still significant, and at intermediate separations it remains undiminished.

The middle panel of Fig. 4 details the effects of using the semiempirical fitting of DFT-D and the larger $C_{6}$ coefficients. We compare the binding curve for cubane obtained by use of vdW-DF with the binding curve obtained with DFT-D calculations. We select the schemes of Grimme $^{17}$ and Jurečka, ${ }^{18}$ as these provide parameters for the PBE flavor of exchange-correlation, which is available to us. ${ }^{80}$

The DFT-D scheme of Grimme scales the strength of the dispersive interaction to a particular semilocal exchangecorrelation to achieve good performance at short separations. For the PBE flavor of GGA, the cohesion energy of a dimer reads

$$
E_{\mathrm{coh}}^{\mathrm{DFT}-\mathrm{D}, \mathrm{G}}=E_{\mathrm{coh}}^{\mathrm{PBE}}+s_{6}^{\mathrm{PBE}} \sum_{i j} f_{\mathrm{PBE}, \mathrm{G}}^{i j}\left(\left|\mathbf{r}_{i}-\mathbf{r}_{j}\right|\right) \frac{C_{6}^{i j}}{\left|\mathbf{r}_{i}-\mathbf{r}_{j}\right|^{6}},
$$

where $s_{6}=0.7$. This scheme therefore sacrifices the asymptotic description in favor of the a good description of binding separations. The scheme of Jurečka instead adjusts parameters of the damping function to the flavor of semilocal exchange-correlation,

$$
E_{\mathrm{coh}}^{\mathrm{DFT}-\mathrm{D}, \mathrm{J}}=E_{\mathrm{coh}}^{\mathrm{PBE}}+\sum_{i j} f_{\mathrm{PBE}, \mathrm{J}}^{i j}\left(\left|\mathbf{r}_{i}-\mathbf{r}_{j}\right|\right) \frac{C_{6}^{i j}}{\left|\mathbf{r}_{i}-\mathbf{r}_{j}\right|^{6}},
$$

and ensure that $f_{\mathrm{PBE}, \mathrm{J}}^{i j} \rightarrow 1$ for large separations. The DFT-D scheme can therefore, in principle, describe both asymptotic and binding separations. However, the question remains on how it performs for systems where characteristic separations lie between these two limits.

The binding curves of vdW-DF(revPBE) (the solid curve) and that for the DFT-D scheme of Grimme(PBE) (upper dash-dotted curve) and Jurečka(PBE) (lower dash-dotted curve) indicate an underestimation of DFT-D at intermediate separations. The minimum at negative $d-d_{0}$ shows that use of DFT-D would improve lattice constants over vdW-DF. ${ }^{54}$ The two DFT-D schemes give quite differing binding energies. Both results show that a binding energy at the same magnitude as vdW-DF can be achieved even with an atombased asymptotic form of the attractive potential. The energy of vdW-DF is significantly larger at intermediate separations than that of Jurečka despite that $C_{6}$ coefficients of asymptotic $v d W-D F$ are underestimated (while those of Jurečka are likely to be somewhat overestimated) and despite that the corresponding DFT-D scheme binds stronger than vdW-DF.

The lower panel of Fig. 4 shows that the difference at intermediate separations comes primarily from the varying accounts of correlation. The solid curve shows the nonlocal correlation of vdW-DF. The dash-dotted upper (lower) curve shows 


$$
\begin{aligned}
E_{\mathrm{app}}^{\mathrm{DFT}-\mathrm{D}, \mathrm{G}(\mathrm{J})}= & \sum_{i j} f_{\mathrm{PBE}, \mathrm{G}(\mathrm{J})}^{i j}\left(\left|\mathbf{r}_{i}-\mathbf{r}_{j}\right|\right) \frac{C_{6}^{i j}}{\left|\mathbf{r}_{i}-\mathbf{r}_{j}\right|^{6}}+\Delta E_{c}^{\mathrm{PBE}}(d) \\
& -\Delta E_{c}^{\mathrm{LDA}}(d),
\end{aligned}
$$

which for DFT-D corresponds best to the nonlocal correlations provided by $\Delta E_{c}^{\mathrm{nl}}$ in vdW-DF. The figure also shows that the difference partly cancels, at short but not at intermediate separations, with the energy difference between the exchange flavors of revPBE and PBE, $\delta E_{x}=\Delta E_{x}^{\mathrm{revPBE}}-E_{x}^{\mathrm{PBE}}$. Thus, for certain exchange functionals, adding an asymptotic atom-based account of nonlocal correlation can generate good binding values, yet our vdW-DF results indicate that this framework is not optimal for describing interactions at intermediate separations.

Our results suggest that APPs and DFT-Ds could be improved at intermediate separations. A possible strategy is to replace the atomic separations $r$ by an effective separation $r-r_{0}$, where $r_{0}$ reflects the image-planes found for nanotubes and surfaces in Refs. 23 and 70. Keeping this (surfacephysics) effect would increase the strength of the dispersion interactions at shorter separations.

In summary, the results of this section suggest that asymptotic atom-based pair potentials has a limited transferability over the full range of separations. Thus, for schemes using such a form, our results raise questions on their ability to generate accurate results under broad condition (having multiple characteristic separations), for instance, involving phase transitions or processes that drive the system out of equilibrium, in protein unfolding, in phase transitions, or simply for systems that have competing interactions. ${ }^{1}$ We note that there is no guarantee that vdW-DF, in its current form, can provide an accurate account under such broad conditions. A vdW-DF limitation is here exemplified by the likely underestimation of $C_{6}$ coefficients for the platonic molecules. Nevertheless, we argue that nonlocal functionals, such as vdW-DF, hold the most promise for dealing with molecular configurations under broad conditions. This is because an electron-based approach provides a framework that naturally includes image-plane and multipole effects. It therefore holds the key to an account that describe the variation in dispersive response over the full range of separations.

\section{CONCLUSIONS}

For the three cagelike organic molecular crystals, hexamine, cubane, and dodecahedrane, vdW-DF gives lattice parameters and cohesion energy that agree well with experiments, although for all three crystals the unit-cell volumes are overestimated. A substantial sensitivity of lattice parameters and cohesion energy to the flavor of semilocal exchange signals that this overestimation stems mostly from the chosen form of exchange functional.

We have also shown that at short and intermediate separations, the full nonlocal correlation of vdW-DF is considerably larger than its corresponding atom-based asymptotic account. Notwithstanding that the asymptotic account of vdW-DF likely needs improvement, this enhancement indi- cates that the asymptotic $1 / r^{6}$ form of atomic pair potentials, by construction, cannot give a transferable account over a large range of separations.

This paper underlines the usefulness of studying simple model sparse systems as molecular crystals to gain insight into methods intended for the study of sparse and supramolecular systems. ${ }^{1}$ Both DFT-D and vdW-DF benefit from such testing because they are designed to be parameter-free, and an accurate account of molecular crystals would suggest an accurate account of more complex assemblies of similar molecules. The presented molecular crystals provide particularly accessible cases of extended systems and can therefore be used in conjunction with future development of pairpotential methods and exchange-correlation functionals.

\section{ACKNOWLEDGMENTS}

We acknowledge E. Schröder for useful comments and discussions. We thank I. Sinno for help in designing the molecular crystal schematics and $\varnothing$. Borck for access to his code for Bader analysis. The Swedish National Infrastructure for Computing (SNIC) is acknowledged for computer allocation and for $\mathrm{KBs}$ participation in the graduate school NGSSC. The work was supported by the Swedish Research Council (Vetenskapsrådet VR) under Grant No. 621-20084346.

${ }^{1}$ J. M. Lehn, Science 260, 1762 (1993).

${ }^{2}$ J. Kleis, B. I. Lundqvist, D. C. Langreth, and E. Schröder, Phys. Rev. B 76, 100201 (2007).

${ }^{3}$ S. H. Vosko, L. Wilk, and M. Nusair, Can. J. Phys. 58, 1200 (1980).

${ }^{4}$ J. P. Perdew and Y. Wang, Phys. Rev. B 45, 13244 (1992).

${ }^{5}$ J. P. Perdew, K. Burke, and M. Ernzerhof, Phys. Rev. Lett. 77, 3865 (1996).

${ }^{6}$ G. M. Day, J. Chisholm, N. Shan, W. D. S. Motherwell, and W. Jones, Cryst. Growth Des. 4, 1327 (2004).

${ }^{7}$ W. Kohn, Y. Meir, and D. E. Makarov, Phys. Rev. Lett. 80, 4153 (1998).

${ }^{8}$ J. F. Dobson and J. Wang, Phys. Rev. Lett. 82, 2123 (1999).

${ }^{9}$ H. Rydberg, M. Dion, N. Jacobson, E. Schröder, P. Hyldgaard, S. I. Simak, D. C. Langreth, and B. I. Lundqvist, Phys. Rev. Lett. 91, 126402 (2003).

${ }^{10}$ M. Dion, H. Rydberg, E. Schröder, D. C. Langreth, and B. I. Lundqvist, Phys. Rev. Lett. 92, 246401 (2004); 95, 109902(E) (2005).

${ }^{11}$ O. A. Vydrov and T. Van Voorhis, J. Chem. Phys. 130, 104105 (2009).

${ }^{12}$ O. A. Vydrov and T. Van Voorhis, Phys. Rev. Lett. 103, 063004 (2009).

${ }^{13}$ M. A. Basanta, Y. J. Dappe, J. Ortega, and F. Flores, Europhys. Lett. 70, 355 (2005).

${ }^{14}$ Y. J. Dappe, M. A. Basanta, F. Flores, and J. Ortega, Phys. Rev. B 74, 205434 (2006).

${ }^{15}$ X. Wu, M. C. Vargas, S. Nayak, V. Lotrich, and G. Scoles, J. Chem. Phys. 115, 8748 (2001).

${ }^{16}$ Q. Wu and W. Yang, J. Chem. Phys. 116, 515 (2002).

${ }^{17}$ S. Grimme, J. Comput. Chem. 25, 1463 (2004).

${ }^{18}$ P. Jurečka, J. Černy, P. Hobza, and D. R. Salahub, J. Comput. Chem. 28, 555 (2007).

${ }^{19}$ V. Barone, M. Casarin, D. Forrer, M. Pavone, M. Sambi, and A. Vittadini, J. Comput. Chem. 30, 934 (2008).

${ }^{20}$ M. A. Neumann and M. Perrin, J. Phys. Chem. B 109, 15531 (2005).

${ }^{21}$ D. C. Langreth, B. I. Lundqvist, S. D. Chakarova-Kack, V. R. Cooper, M. Dion, P. Hyldgaard, A. Kelkkanen, J. Kleis, L. Kong, S. Li, P. G. Moses, E. Murray, A. Puzder, H. Rydberg, E. Schrder, and T. Thonhauser, J. Phys.: Condens. Matter 21, 084203 (2009).

${ }^{22}$ V. R. Cooper, T. Thonhauser, A. Puzder, E. Schröder, B. I. Lundqvist, and D. C. Langreth, J. Am. Chem. Soc. 130, 1304 (2008).

${ }^{23}$ J. Kleis, E. Schröder, and P. Hyldgaard, Phys. Rev. B 77, 205422 (2008).

${ }^{24}$ A. Kelkkanen, B. I. Lundqvist, and J. K. Nørskov, J. Chem. Phys. 131, 046102 (2009).

${ }^{25}$ L. Kong, G. Román-Pérez, J. M. Soler, and D. C. Langreth, Phys. Rev. 
Lett. 103, 096103 (2009)

${ }^{26}$ P. Sony, P. Puschnig, D. Nabok, and C. Ambrosch-Draxl, Phys. Rev. Lett. 99, 176401 (2007).

${ }^{27}$ N. Atodiresei, V. Caciuc, P. Lazic, and S. Blügel, Phys. Rev. Lett. 102, 136809 (2009).

${ }^{28}$ K. Berland, T. L. Einstein, and P. Hyldgaard, Phys. Rev. B 80, 155431 (2009).

${ }^{29}$ K. Toyoda, Y. Nakano, I. Hamada, K. Lee, S. Yanagisawa, and Y. Morikawa, Surf. Sci. 603, 2912 (2009).

${ }^{30}$ A. Gavezzotti, Modell. Simul. Mater. Sci. Eng. 10, R1 (2002).

${ }^{31}$ T. Yildirim, P. M. Gehring, D. A. Neumann, P. E. Eaton, and T. Emrick, Phys. Rev. Lett. 78, 4938 (1997).

${ }^{32}$ Plato, Timaeus, http://classics.mit.edu/Plato/timaeus.html.

${ }^{33}$ P. E. Eaton and T. W. Cole, J. Am. Chem. Soc. 86, 3157 (1964).

${ }^{34}$ L. A. Paquette, R. J. Ternansky, D. W. Balogh, and G. J. Kentgen, J. Am. Chem. Soc. 105, 5446 (1983).

${ }^{35}$ M. N. Glukhovtsev, S. Laiter, and A. Pross, J. Phys. Chem. 99, 6828 (1995).

${ }^{36}$ M. S. Ahmed, Acta Crystallogr. 5, 587 (1952).

${ }^{37}$ R. Rao, T. Sakuntala, S. K. Deb, A. P. Roy, V. Vijayakumar, B. K. Godwal, and S. K. Sikka, Chem. Phys. Lett. 313, 749 (1999).

${ }^{38}$ G. H. Heilmeier, Appl. Opt. 3, 1281 (1964).

${ }^{39}$ R. G. Dickinson and A. L. Raymond, J. Am. Chem. Soc. 45, 22 (1923).

${ }^{40}$ B. S. Hudson, D. G. Allis, S. F. Parker, A. J. Ramirez-Cuesta, H. Herman, and H. Prinzbach, J. Phys. Chem. A 109, 3418 (2005).

${ }^{41}$ A. Alamdari and F. Tabkhi, Chem. Eng. Prog. 43, 803 (2004).

${ }^{42}$ Ullmann's Encyclopedia of Industrial Chemistry, 2008.

${ }^{43}$ S. Borman, Chem. Eng. News 72, 34 (1994).

${ }^{44}$ E. Ziambaras and E. Schröder, Phys. Rev. B 68, 064112 (2003).

${ }^{45}$ T. Thonhauser, V. R. Cooper, S. Li, A. Puzder, P. Hyldgaard, and D. C. Langreth, Phys. Rev. B 76, 125112 (2007).

${ }^{46}$ D. C. Langreth, M. Dion, H. Rydberg, E. Schröder, P. Hyldgaard, and B. I. Lundqvist, Int. J. Quantum Chem. 101, 599 (2005).

${ }^{47}$ E. Ziambaras, J. Kleis, E. Schröder, and P. Hyldgaard, Phys. Rev. B 76, 155425 (2007).

${ }^{48}$ Y. Zhang and W. Yang, Phys. Rev. Lett. 80, 890 (1998).

${ }^{49}$ W. Kohn and L. Sham, Phys. Rev. 140, A1133 (1965).

${ }^{50}$ G. Román-Pérez and J. M. Soler, Phys. Rev. Lett. 103, 096102 (2009).

${ }^{51}$ S. Li, V. R. Cooper, T. Thonhauser, A. Puzder, and D. C. Langreth, J. Phys. Chem. A 112, 9031 (2008)

${ }^{52}$ A. Puzder, M. Dion, and D. C. Langreth, J. Chem. Phys. 124, 164105 (2006).

${ }^{53}$ A. Gulans, M. J. Puska, and R. M. Nieminen, Phys. Rev. B 79, 201105 (2009)

${ }^{54}$ Several new exchange flavors designed for use within the vdW-DF framework promises to improve the vdW-DF account of binding separations: V. R. Cooper, e-print arXiv:0910.1250v1; J. Klimeš, D. R. Bowler, and A. Michaelides, J. Phys.: Condens. Matter 22, 022201 (2010).

${ }^{55}$ S. D. Chakarova-Käck, E. Schröder, B. I. Lundqvist, and D. C. Langreth,
Phys. Rev. Lett. 96, 146107 (2006).

${ }^{56}$ Opensource code DACAPO, http://www.fysik.dtu.dk/CAMPOS/

${ }^{57}$ L. N. Becka and D. W. J. Cruickshank, Proc. R. Soc. London, Ser. A 273 , 435 (1963).

${ }^{58}$ L. Hedberg, K. Hedberg, P. E. Eaton, N. Nodari, and A. G. Robiette, J. Am. Chem. Soc. 113, 1514 (1991).

${ }^{59}$ M. Bertau, F. Wahl, A. Weiler, K. Scheumann, J. Worth, M. Keller, and H. Prinzbach, Tetrahedron 53, 10029 (1997).

${ }^{60}$ E. A. Arnautova, M. V. Zakharova, T. S. Pivina, E. A. Smolenskii, D. V. Sukhachev, and V. V. Shcherbukhin, Russ. Chem. Bull. 45, 2723 (1996).

${ }^{61}$ G. N. Ramachandran and W. A. Wooster, Acta Crystallogr. 4, 431 (1951).

${ }^{62}$ J. S. Chickos and W. E. Acree, J. Phys. Chem. Ref. Data 31, 537 (2002).

${ }^{63}$ J. C. Galluci, C. W. Doecke, and L. A. Paquette, J. Chem. Phys. 108, 1343 (1986)

${ }^{64}$ D. Nabok, P. Puschnig, and C. Ambrosch-Draxl, Phys. Rev. B 77, 245316 (2008).

${ }^{65}$ A. T. Hagler, E. Huler, and S. Lifson, J. Am. Chem. Soc. 96, 5319 (1974).

${ }^{66}$ W. F. Perger, J. Criswell, B. Civalleri, and R. Dovesi, Comput. Phys. Commun. 180, 1753 (2009).

${ }^{67}$ T. Yildirim, S. Ciraci, Ç. Kiliç, and A. Buldum, Phys. Rev. B 62, 7625 (2000).

${ }^{68}$ H. Margenau and N. R. Kestner, Theory of Interatomic Forces, 2nd ed. (Pergamon, Oxford, 1969).

${ }^{69}$ G. Mahan, J. Chem. Phys. 43, 1569 (1965).

${ }^{70}$ E. Zaremba and W. Kohn, Phys. Rev. B 13, 2270 (1976).

${ }^{71}$ R. F. W. Bader, Atoms in Molecules: A Quantum Theory (Oxford University Press, Oxford, 1990).

${ }^{72}$ G. Henkelman, A. Arnaldsson, and H. Jónsson, Comput. Mater. Sci. 36, 354 (2006).

${ }^{73}$ Y. Andersson, D. C. Langreth, and B. I. Lundqvist, Phys. Rev. Lett. 76, 102 (1996).

${ }^{74}$ We have explicitly verified that the precise assignment of the total molecular response onto the atoms does not alter the APP-vdWDF curves significantly. An assignment of the total asymptotic vdW-DF response according to the relative strength of Grimme yields a weak increase in the difference between APP-vdWDF and the nonlocal correlation.

${ }^{75}$ J. C. Slater and J. G. Kirkwood, Phys. Rev. 37, 682 (1931).

${ }^{76}$ Y. Andersson and H. Rydberg, Phys. Scr. 60, 211 (1999).

${ }^{77}$ M. Pavone, N. Rega, and V. Barone, Chem. Phys. Lett. 452, 333 (2008).

${ }^{78}$ C. Morgado, M. A. Vincent, I. H. Hillier, and X. Shan, Phys. Chem. Chem. Phys. 9, 448 (2007)

${ }^{79}$ S. Grimme, J. Antony, T. Schwabe, and C. Mck-Lichtenfeld, Org. Biomol. Chem. 5, 741 (2007)

${ }^{80}$ In our comparison, we neglect that these schemes are optimized for a particular basis set, arguing that our comparison does not require a finetuned version of DFT-D. 